\title{
Relating Retinal Nerve Fiber Layer Thickness and Functional Estimates of Ganglion Cell Sampling Density in Healthy Eyes and in Early Glaucoma
}

\author{
Tony Redmond, ${ }^{* 1,2}$ Roger S. Anderson, ${ }^{1,2}$ Richard A. Russell, ${ }^{1,3}$ and David F. Garway-Heath ${ }^{1,3}$
}

Purpose. To investigate the relationship between retinal nerve fiber layer (RNFL) thickness and peripheral grating resolution acuity (PGRA) as well as differential light sensitivity (DLS) in healthy subjects and patients with early glaucoma. The agreement between estimates of retinal ganglion cell (GC) density from each functional test is explored.

Methods. PGRA was measured in 24 patients with early glaucoma (mean deviation $[\mathrm{MD}]>-8 \mathrm{~dB}$ ) and 26 healthy subjects using achromatic Gabor stimuli in 4 diagonal visual field locations at $10^{\circ}$ eccentricity. DLS for a Goldmann size III equivalent was obtained from individual spatial summation functions and expressed in Humphrey Field Analyzer-equivalent decibel values. RNFL thickness was measured around the optic nerve head using Zeiss Stratus optical coherence tomography and related to functional measures using a retinotopic map. Functional GC density was estimated using structure/function models for both tests. Passing-Bablok regression was used to investigate the structure/function relationships.

REsults. A positive and statistically significant association was found between PGRA and RNFL thickness, and separately between DLS and RNFL thickness, for combined glaucoma and healthy data (both $P<0.05$ ). The slope of the structure/function association in healthy subjects was not significantly different to that in glaucoma patients using either functional measure (both $P>0.05$ ). Agreement

From the ${ }^{1}$ National Institute for Health Research (NIHR) Biomedical Research Centre at Moorfields Eye Hospital National Health Service (NHS) Foundation Trust and University College London (UCL) Institute of Ophthalmology, London, United Kingdom; the ${ }^{2}$ Vision Science Research Group, School of Biomedical Sciences, University of Ulster, Cromore Road, Coleraine, Northern Ireland, United Kingdom; the ${ }^{3}$ Division of Optometry and Visual Sciences, City University London, Northampton Square, London, United Kingdom.

Supported by a PhD scholarship from the Department of Employment and Learning, Northern Ireland (TR), and in part by the Department of Health National Institute for Health Research (NIHR) Biomedical Research Centre at Moorfields Eye Hospital NHS Foundation Trust and UCL Institute of Ophthalmology (TR, RSA, RAR, DFG-H). Also supported by funding from the International Glaucoma Association (DFG-H's chair at UCL).

Submitted for publication June 5, 2012; revised September 27, 2012 and January 30, 2013; accepted February 15, 2013.

Disclosure: T. Redmond, None; R.S. Anderson, None; R.A. Russell, None; D.F. Garway-Heath, Carl Zeiss Meditec (F), Heidelberg Engineering (F)

Current affiliation: "Department of Optometry and Vision Sciences, Cardiff University, Cardiff, United Kingdom.

Corresponding author: Tony Redmond, Department of Optometry and Vision Sciences, Cardiff University, Maindy Road, Cardiff, CF24 4LU, United Kingdom; RedmondT1@cardiff.ac.uk. between estimates of GC density from psychophysical data was moderate.

Conclusions. The relationship between PGRA and RNFL thickness is at least as great in magnitude as that between DLS and RNFL thickness; a significant structure/function association is also observed in healthy subjects alone. (Invest Opbthalmol Vis Sci. 2013;54:2153-2162) DOI:10.1167/ iovs.12-10342

The nature of the association between retinal structure and 1 visual function has been a subject of particular interest in both basic and clinical research for many years. Specifically, a large body of research in the field of ophthalmology has been directed toward the relationship between visual field sensitivity using standard clinical instruments and measurements of retinal nerve fiber layer (RNFL) thickness by optical coherence tomography (OCT) in glaucoma. Reconciliation of measures of retinal structure and function in glaucoma is important for improved understanding of a patient's stage of disease, identifying further deterioration, and determining the effectiveness of treatment with greater confidence in individual patients, especially considering the sizeable variability that accompanies commonly used functional ${ }^{1-3}$ and structural ${ }^{4,5}$ tests. The relationship between retinal structure and sensitivity (with standard automated perimetry [SAP]) has been explored at length. ${ }^{6-12}$ It is clear that the structure/function relationship is not a simple one, and the elucidation of the precise relationship at the retinal level requires consideration of factors such as variability accompanying both structural, and subjective functional tests, measurement scale (i.e., linear or logarithmic), ,6,10,13,14 spatial summation, ${ }^{6,10,15,16}$ and interindividual physiologic variation (e.g., RNFL stratification and the extent of nonneural components of the RNFL).

The findings from some studies of a weak relationship between structure and function in glaucoma (using SAP as the functional test) have led to concerns about false negative diagnoses (missed cases). This has increased interest in alternative forms of perimetry, ${ }^{17-20}$ purported to tap into, and test, specific parallel visual pathways thought to be more vulnerable to glaucoma. The specificity of the different forms of perimetry and the selective vulnerability of the different pathways in glaucoma continue to be deliberated in contemporary literature. ${ }^{21,22}$ Meanwhile, however, there is still merit in attempting to characterize obstacles to the reconciliation of SAP sensitivity and structural measures of the retina, since SAP remains the gold standard clinical test of the visual field.

Peripheral grating resolution acuity (PGRA) is hypothesized to be limited by localized functional ganglion cell (GC) sampling density, ${ }^{23-27}$ and it has been argued that localized measures of grating resolution acuity may be useful in the detection and monitoring of retinal diseases such as glauco- 
ma. ${ }^{15,28,29}$ Based on Shannon's sampling theorem (see Ref. 30 for a review), GC density (D), assuming a hexagonal array, can be related to the minimum angle of resolution (MAR) by the equation MAR $=0.93 / \sqrt{ } \mathrm{D}$ (see Ref. 27 for a derivation of this formula). Sampling theory is well developed generically and has been applied to glaucoma, but the relationship between PGRA and RNFL thickness in healthy or glaucomatous eyes has not yet been explored. Since a strong association has been shown between PGRA in healthy eyes and estimates of grating resolution acuity from previously published counts of normal GC density, ${ }^{26}$ the possibility exists that PGRA relates more closely than SAP to clinical measures of retinal structure. Furthermore, direct comparisons of PGRA and SAP sensitivity are lacking and no studies to date have reported the agreement between estimates of functional GC density from either functional test.

The main aims of this study are (1) to investigate the relationship between PGRA and RNFL thickness, as measured with OCT, (2) to investigate the relationship between differential light sensitivity (DLS) and RNFL thickness, (3) to explore the relationship between RNFL thickness and estimates of underlying functional GC density derived from the functional measurements using established structure/function models, and (4) to explore the agreement between both estimates of functional GC density. The primary hypothesis is that PGRA and RNFL thickness are associated in healthy and glaucomatous eyes. Secondary hypotheses are that (1) DLS and RNFL thickness are associated in healthy and glaucomatous eyes, (2) there is an association between PGRA and RNFL thickness and between DLS and RNFL thickness in healthy subjects alone, (3) the slope of each structure/function relationship (with each functional measure) in healthy subjects is not significantly different to that observed in glaucoma patients, (4) the slope of the PGRA/RNFL thickness association is not significantly different to that of the DLS/RNFL thickness association, (5) there is an association between PGRA and DLS in healthy and glaucomatous eyes, and (6) the slope of the relationship between functional GC density and RNFL thickness is steeper when PGRA, rather than DLS, is used to estimate GC density.

\section{Methods}

\section{Subjects}

Twenty-four white European individuals with early glaucoma (mean age: 63 years, range, $46-78$ years) and 26 white European, age-similar healthy subjects (mean age: 62 years, range, $51-77$ years) were tested as part of a cross-sectional prospective study. ${ }^{31}$ One eye was tested per individual. All patients were previously diagnosed with POAG; 13 with high-tension and 11 with normal-tension glaucoma. Each participant underwent a full eye examination, including retinoscopy and subjective refraction. Each was required to have a best-corrected visual acuity of at least $6 / 9(20 / 30)$, refractive error less than \pm 6.00 diopters in any meridian and less than 1.25 diopters of astigmatism, clear media, a controlled IOP less than $21 \mathrm{~mm} \mathrm{Hg}$, and no other abnormal ocular or systemic condition considered to affect visual performance. All participants were required to have undergone at least two reliable visual field tests ( $<33 \%$ fixation losses, $<33 \%$ false negative responses, and $<20 \%$ false positive responses) within 6 months prior to experiments. Patients were recruited on the basis of a rim area outside normal limits. Each glaucoma patient had a mean deviation (MD) better than $-8 \mathrm{~dB}$ on SAP (Humphrey Field Analyser, HFA II, SITA 24-2 test pattern; Carl Zeiss Meditec, Inc., Dublin, CA). In patients, the average total deviation for the SAP locations $( \pm 3, \pm 9)$ nearest the experimental test locations was $-1.3 \mathrm{~dB}$ (range, $+2 \mathrm{~dB}$ to $-8 \mathrm{~dB}$ ). Healthy individuals were included if they had no family history of glaucoma, a full visual field (including a report of within normal limits on the glaucoma hemifield test) and on the basis of a rim area within normal limits on a Heidelberg Retina Tomograph II (Heidelberg Engineering, Heidelberg, Germany) with Moorfields Regression Analysis. A favorable ethical opinion was given by the Oxfordshire National Health Service Research Ethics Committee A and the Moorfields Eye Hospital Research Governance Committee. This research was conducted in accordance with the tenets of the Declaration of Helsinki.

\section{Determination of Retinal Nerve Fiber Layer Thickness}

Peripapillary RNFL thickness was measured with time-domain OCT (Zeiss Stratus Optical Coherence Tomographer; Carl Zeiss Meditec, Inc.) and the circular 3.4-mm Fast RNFL scan protocol. Three scans were acquired and point wise RNFL thickness values were averaged. Only images of good quality were included; quality was assessed by scan circle alignment in relation the optic nerve head (a subjective judgment by a clinician experienced in OCT scan acquisition), the number of good A-scans ( $>95 \%$ ), signal-to-noise ratio greater than 32, signal strength greater than 7 , and algorithm confidence greater than 7 . Images were reacquired if quality was initially inadequate for inclusion. Each image was individually inspected for any irregularities caused by the presence of blood vessels in the sectors under analysis, however, none were found.

\section{Functional Tests: Apparatus and Stimuli}

PGRA was measured as part of a previous study by the authors. ${ }^{31}$ Gabor stimuli were generated on a $\gamma$-corrected 21-inch greyscale monitor (Phillips Fimi MGD-403, pixel resolution: $1280 \times 965$, frame rate: $73 \mathrm{~Hz}$; Ampronix, Irvine, CA) with a visual stimulus generator (VSG; Cambridge Research Systems, Ltd., Rochester, UK). Stimuli were static (nonflickering) with a maximum contrast of $90 \%$ and a SD of $1.5^{\circ}$, with their centers placed at $10^{\circ}$ eccentricity from the fovea at four separate meridians centered on the fovea $\left(36^{\circ}, 144^{\circ}\right.$, $216^{\circ}$, and $324^{\circ}$ ). Vertical and horizontal gratings were chosen as the alternatives for the resolution task as they are orientated obliquely with respect to the meridian and, thus, display comparable acuity. ${ }^{32,33}$ Stimuli were presented on a grey background of $10 \mathrm{~cd} /$ $\mathrm{m}^{2}$. The mean stimulus luminance was equal to the luminance of the achromatic background. Total stimulus duration was 1 second, including a $300 \mathrm{~ms}$ stimulus onset and $300 \mathrm{~ms}$ decay. A white fixation cross was centered on the screen. The viewing distance was $102 \mathrm{~cm}$.

DLS values for circular increments were derived from individual spatial summation functions of the same subjects in the previous study, ${ }^{31}$ in an attempt to minimize the error in the functional measure. In that study, spatial summation functions were measured for six circular incremental stimuli of different size on the same apparatus. Stimulus duration was $200 \mathrm{~ms}$. Stimulus configuration was the same as that used in SAP, except that stimulus area (range, $0.01-2.67 \mathrm{deg}^{2}$ ) was not required to match those of the Goldmann stimuli. Test locations were identical between the two psychophysical tests. An initial practice run was conducted, which lasted until the participant fully understood the test.

\section{Correction of Refractive Error}

A refractive correction was given where appropriate. Calculation of the appropriate refractive correction for the test locations is described in the previous study. ${ }^{34}$ Refractive error did not differ between test locations for any subject and a single refractive correction was given for all test locations. Test-retest variability was no greater than 0.25 diopters for any subject. 


\section{Psychophysical Procedure}

The procedure for the PGRA test is described in full in the previous study, ${ }^{34}$ however, the main points are described below. One drop of tropicamide hyrochloride (1\%) was instilled in the eye under test, 20 minutes before the commencement of experiments. A two-alternative forced choice (2AFC) test was used to determine PGRA, whereby the observer was required to press one of two buttons on a response box to indicate whether he/she perceived the grating to be orientated vertically or horizontally. All four visual field locations were tested during the same run, in an interleaved fashion. Observers were encouraged to guess the orientation, should the grating become unresolvable or undetectable. A 3-up/1-down staircase procedure was employed, using equal upward and downward steps of $0.04 \log$ units (converging on the $79 \%$ seen level ${ }^{35}$ ). An initial practice run was conducted that lasted until two reversals had been reached and the participant fully understood the task. Threshold for the experimental test was recorded as the average from four reversals, in cycles/deg. False negative responses were tested periodically by presenting gratings with a spatial frequency that was easily resolvable at that location (as determined prior to data collection). Fixation was monitored visually. For PGRA, results were considered unreliable and subsequently excluded if the false negative rate exceeded $30 \%$ or if the subject made noticeable saccades throughout the experiment. The order of tests was randomized for each observer.

For the detection task, thresholds were determined separately, but in a random order, for each of the six differently sized stimuli, with a best-PEST (Parameter Estimation by Sequential Testing) adaptive algorithm ${ }^{36}$ and a Yes/No response criterion.

\section{Analysis}

A two-phase regression model ${ }^{37}$ was fitted to the area/threshold (spatial summation) data from the superior and inferior hemifields of each subject in the previous study. ${ }^{31} \mathrm{~A}$ full description of the analysis of the area/threshold data is given in that study. Increment thresholds for a stimulus equivalent in size to a Goldmann III stimulus were determined from each curve and were converted to increment luminance values ( $\mathrm{L}$, in $\mathrm{cd} / \mathrm{m}^{2}$ ). HFA II stimulus luminance (in $\mathrm{cd} /$ $\mathrm{m}^{2}$ ) is related to its difference (in decibels) from the maximum luminance of the instrument (i.e., its corresponding sensitivity level) by the equation

$$
\text { DLS }=-4.34 \times \ln (L)+35
$$

HFA II-equivalent DLS values (in decibels) were determined from increment luminance values in this way.

Arithmetic average values for PGRA and DLS were calculated (i.e., values were converted to linear units, averaged, and converted back to logarithmic units, where required) for the superior and inferior hemifield for each observer.

In order to compare structure and function, nerve fiber layer bundles underlying the center of each PGRA/DLS test location were traced back to the optic nerve head using a retinal photograph from an original average observer in the study of Garway-Heath et al. ${ }^{38}$ Average RNFL thickness was calculated for $30^{\circ}$ sectors centered on the point of incidence of the nerve fiber trace on the scan circle. Superior and inferior sectors were averaged separately so that each individual had one superior and one inferior peripapillary measurement. Superior and inferior sector data will, herein, be referred to in relation to their corresponding visual field regions for ease of discussion (i.e., superior/ inferior hemifield).

\section{Estimating Functional Ganglion Cell Density}

It is challenging to compare structure/function relationships directly when functional measures are not represented on common scales. Thus, estimates of GC density, yielded by structure/function models relating to the two functional tests, were determined and compared with RNFL thickness.

GC density was estimated from PGRA values, assuming a hexagonal array. PGRA was first converted to MAR and multiplied by 0.275 to convert angular distance to distance in millimeters on the retina. ${ }^{39}$ The equation of Thibos $^{27}$ was rearranged to estimate the number of GCs per millimeter squared:

$$
\mathrm{GC} / \mathrm{mm}^{2}(\mathrm{PGRA})=\left(\frac{0.93}{M A R}\right)^{2}
$$

DLS values were converted to underlying GC number using the 'hockey-stick' model of Swanson et al. ${ }^{10}$ as follows:

$$
\mathrm{GC} / \mathrm{mm}^{2}(\mathrm{DLS})=\left\{\begin{array}{lll}
\frac{10^{0.1 \times(D L S-16)}}{a_{r}} & \text { if } & D L S<31 d B \\
\frac{10^{0.4 \times(D L S-27.44)}}{a_{r}} & \text { if } & D L S>31 d B
\end{array}\right.
$$

where $a_{r}=0.0109$, the area of a Goldmann III stimulus at the retina in millimeters squared, using the degrees/millimeter conversion factor of Drasdo and Fowler. ${ }^{39}$ The numerator describes the relationship between DLS and the number of ganglion cells per $0.145 \mathrm{deg}^{2}$ (the area of a Goldmann III stimulus). The equation incorporates a conversion of decibel values to linear units. The slope and intercept of the steep portion of the model (in decibels) are 10 and 16, respectively, while those of the shallow portion are 2.5 and 27.44, respectively. Estimates that exceeded $10,000 \mathrm{GC} / \mathrm{mm}^{2}$ (only two data points) were regarded as outliers (since this number is almost double that found by Dacey ${ }^{25}$ for midget cells at this eccentricity) and were removed from further analysis.

\section{Comparing Structure and Function}

All structure/function and function/function relationships were described using Passing-Bablok regression, ${ }^{40,41}$ as were the associations between RNFL thickness and functional GC density. Unlike ordinary least squares linear regression, Passing-Bablok regression does not assume that either variable is free from error, nor does it make any assumptions about the distribution of the data, and since this analysis is nonparametric, it may be less influenced by outliers. Additionally, while other methods that minimize residuals orthogonal to the regression line (e.g., Deming regression) require a predefined variance ratio to be specified in the model, Passing-Bablok regression may be more useful when such information is unavailable. In short, the regression technique reports the median slope and intercept from an ordered distribution of pair-wise slopes from the dataset. Nonlinear samples are not suitable for Passing-Bablok regression so nonlinearity was tested using a cumulative sum (cusum) test in advance of PassingBablok regression analysis in the current study. Passing and Bablok ${ }^{42}$ recommended that if one rejects the null hypothesis for this test, then this method of regression should not be performed. A more comprehensive explanation of the procedure is given by Bablok et al. ${ }^{41}$ Where data are in logarithmic units, reference herein to nonlinearity, or otherwise, refers to the nature of the relationship on a $\log -\log$ scale. The strength of function/function, structure/function, and RNFL thickness/GC density associations was described by Kendall's $\tau$ and 95\% confidence intervals (CIs) were determined with a bootstrap method, where appropriate.

Permutation analysis was used to test the statistical significance of the structure/function relationships and of differences in structure/ function and function/function slopes between patients and healthy subjects. The observed test statistic was calculated as the difference between slopes for the two associations under test (e.g., between separate data for glaucoma patients and healthy subjects). Each pair of variables was randomly reassigned to one of two groups, matched in sample size with the test data, without replacement. Regression analysis was performed again for the reassigned groups and the 

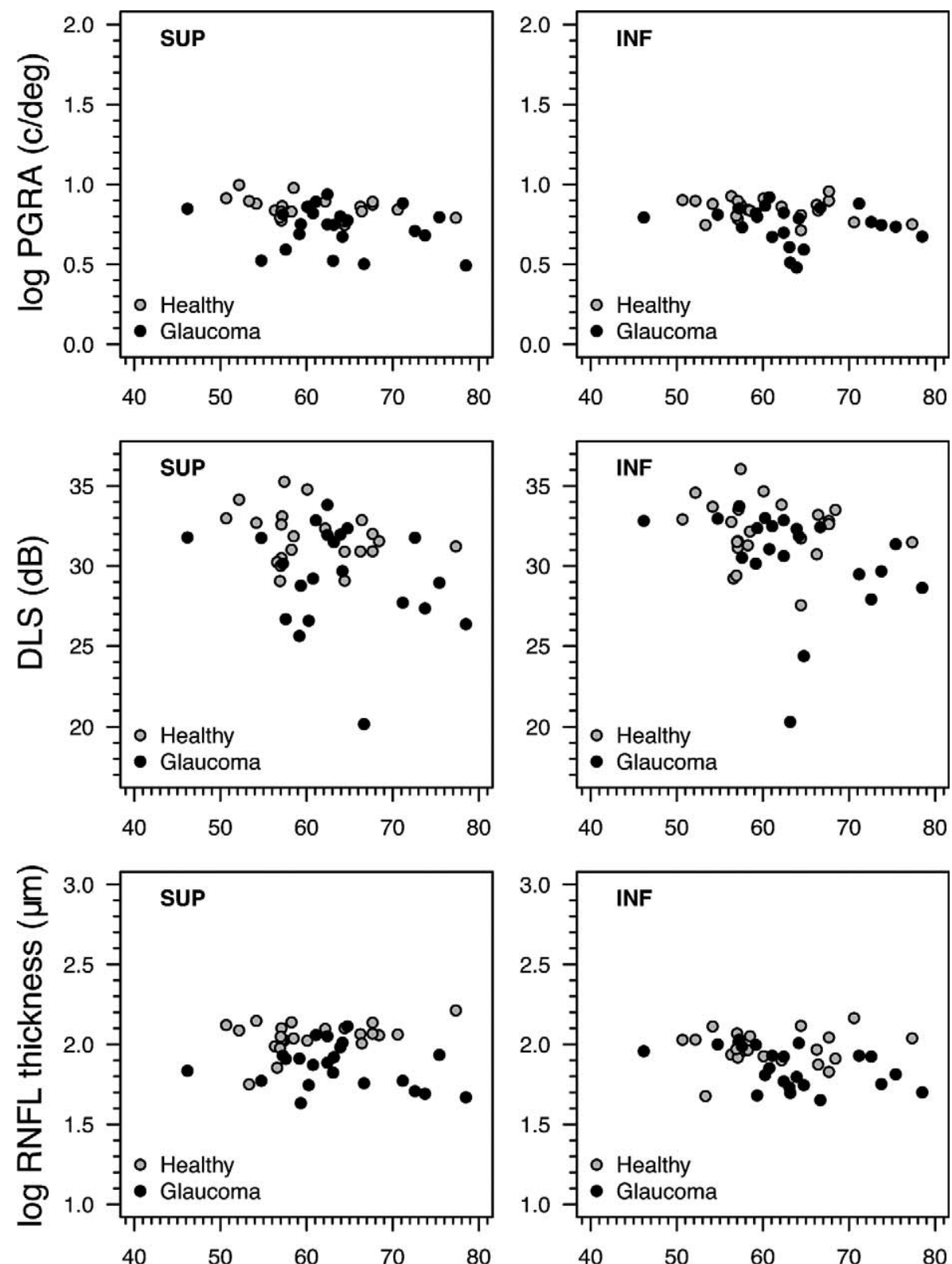

Age (years)

Figure 1. PGRA (top), DLS (middle), and RNFL thickness (bottom) for glaucoma patients and healthy subjects in the superior and inferior visual field.

difference between slopes was calculated. This procedure was performed 5000 times and a distribution of differences between slopes was compiled. A two-sided $P$ value was determined for the observed test statistic, based on its position in the distribution.

Agreement between estimates of functional GC density using PGRA and DLS was assessed with Bland-Altman analysis. ${ }^{43}$

In the current study, the significance level was set at 0.05. A Holm-Bonferroni correction ${ }^{44}$ was applied where there were multiple tests of the same hypothesis (e.g., when testing the same hypothesis in both the superior and inferior hemifield). Where $P$ greater than 0.05 is reported and there are multiple tests of the same hypothesis, this indicates that the effect is not statistically significant at the target level of 0.05. A correction was not applied to the cusum test, as this was applied only to test the assumptions of PassingBablok regression in this study. All statistical analyses were carried out using the freely available, open source statistical environment, $R^{45}$

\section{Results}

Six spatial summation curves were excluded from analysis in the previous study ${ }^{31}$ on the basis of a poor fit $\left(r^{2}<0.9\right)$ of the two-phase regression line and were, thus, excluded from analysis as part of the current study also. PGRA measurements were unreliable for 3 of the 50 subjects, owing to fixation losses, and these were also excluded from further analysis. Although the false negative rate was required to be less than $33 \%$ for PGRA, it did not exceed 5\% for any subject.

Figure 1 shows the degree of overlap of patient and healthy subject data, for reference. 

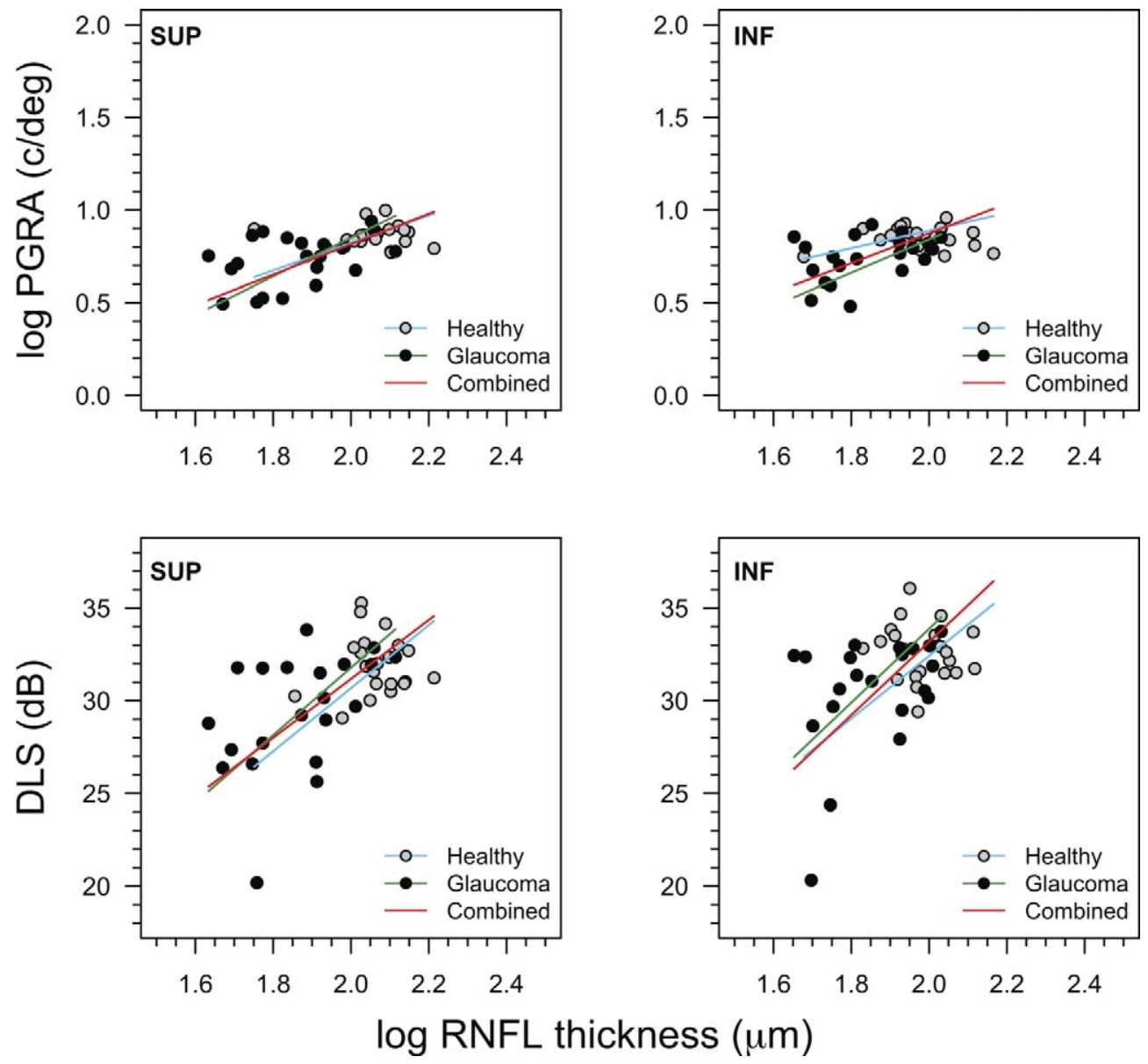

FIGURE 2. Upper panels: the association between PGRA and RNFL thickness for glaucoma patients and healthy subjects in the superior and inferior hemifields. Lower panels: the relationship between DLS and log RNFL thickness for glaucoma patients and healthy subjects in the superior and inferior hemifields. Passing-Bablok slopes are shown in each panel.

\section{The Structure/Function Relationship}

The results of Passing-Bablok regression of PGRA on RNFL thickness, and of DLS on RNFL thickness (both on log-log scales), are shown in Figure 2. Regression lines are shown for glaucoma and healthy data separately, as well as for combined data. On inspection of the data for glaucoma patients, healthy subjects, and both groups combined, there is no apparent nonlinearity when either functional test is used. The absence of statistically significant nonlinearity in these data (cusum, $P>$ 0.05) supports this observation. A positive association was found between PGRA and RNFL thickness, and separately between DLS and RNFL thickness, for combined glaucoma and healthy data. The Holm-Bonferroni-adjusted CIs for these slopes did not include zero for either hypothesis test. A positive association was found between PGRA and RNFL thickness, and separately between DLS and RNFL thickness, in healthy subjects alone. Here too, the Holm-Bonferroni-adjusted CIs for the slopes did not include zero for either hypothesis test. Furthermore, visual inspection of each subplot in Figure 2 suggests that, for either functional test, the slope and intercept of the regression line in healthy subject data alone are similar to those in glaucoma patient data alone. Indeed, the difference between PGRA/RNFL thickness slopes for patients and healthy subjects was not statistically significant using the permutation analysis outlined in the Methods section $(P>0.05$; adjusted 95\% confidence limits for the difference between slopes: superior $-0.56,0.55$; inferior $-0.55,0.57$; Fig. 2). The difference between DLS/RNFL thickness slopes for patients and healthy subjects was not statistically significant either $(P>$ 0.05 ; adjusted $95 \%$ confidence limits for the difference between slopes: superior $-1.88,1.9$; inferior -1.27 , 1.23; Fig. 2). The slope of the DLS/RNFL association appeared steeper than that of the PGRA/RNFL association for combined data on a $\log$-log scale, but this difference was not statistically significant $(P>0.05$; adjusted $95 \%$ confidence limits for the difference between slopes: superior $-4.02,3.91$; inferior $-4.63,4.68)$. The strength of the associations in combined glaucoma and healthy subject data was moderate (Fig. 2) and appeared similar to each other for the range of damage studied here.

\section{The Function/Function Relationship}

Passing-Bablok slopes of the function/function association in glaucoma patients, healthy subjects, and combined groups are given in the Table along with their Holm-Bonferroni-adjusted 95\% CIs. Regression lines were fitted to the data in Figure 3. The relationship between PGRA and DLS, pooled across patients and healthy subjects, showed no significant deviation from linearity (cusum, $P>0.05$ ). This was also the case in the glaucoma and healthy subject groups separately (cusum, $P>$ 0.05). In Figure 3 , the slope of the function/function relationship in healthy subjects appears to be similar to that in glaucoma patients alone. The difference in slopes was not statistically significant, with permutation analysis $(P>0.05)$ (Table, Fig. 3). The strength of the relationship was moderate 
TABLE. Passing-Bablok Slopes (with Adjusted 95\% CI) for Function/Function and Structure/Function Relationships

\begin{tabular}{|c|c|c|c|c|c|c|c|c|}
\hline \multirow[b]{2}{*}{ Test } & \multicolumn{4}{|c|}{ Superior } & \multicolumn{4}{|c|}{ Inferior } \\
\hline & Slope & Adjusted 95\% CI & Kendall's $\tau$ & $P$ Value* & Slope & Adjusted 95\% CI & Kendall's $\tau$ & $P$ Value* \\
\hline \multicolumn{9}{|l|}{ (a) } \\
\hline DLS/RNFL & 1.59 & 1.0 to 2.82 & 0.35 & 0.001 & 1.98 & 0.89 to 3.69 & 0.19 & 0.07 \\
\hline PGRA/RNFL & 0.82 & 0.51 to 1.24 & 0.36 & $<0.001$ & 0.80 & 0.46 to 1.42 & 0.20 & 0.05 \\
\hline PGRA/DLS & 0.40 & 0.27 to 0.65 & 0.42 & $<0.001$ & 0.37 & 0.27 to 0.55 & 0.41 & $<0.001$ \\
\hline \multicolumn{9}{|l|}{ (b) } \\
\hline DLS/RNFL & 1.70 & 0.30 to 11.17 & & & 1.69 & 0.24 to 8.54 & & \\
\hline PGRA/RNFL & 0.74 & 0.28 to 3.59 & & & 0.47 & 0.05 to 2.55 & & \\
\hline PGRA/DLS & 0.41 & 0.17 to 0.81 & & & 0.35 & 0.15 to 0.57 & & \\
\hline \multicolumn{9}{|l|}{ (c) } \\
\hline DLS/RNFL & 1.82 & 0.91 to 4.27 & & & 1.99 & 0.66 to 5.97 & & \\
\hline PGRA/RNFL & 1.04 & 0.45 to 2.14 & & & 0.90 & 0.43 to 2.20 & & \\
\hline PGRA/DLS & 0.40 & 0.20 to 1.06 & & & 0.35 & 0.22 to 0.74 & & \\
\hline
\end{tabular}

Slope values are for log-log data. Kendall's $\tau$ and its associated $P$ value are shown alongside slopes and adjusted CIs for combined glaucoma/ healthy subject data in (a). Slopes and adjusted CIs for separate healthy and glaucoma data are given in (b) and (c), respectively. RNFL thickness data were converted to their corresponding visual field regions for analysis.

$*$ alpha $=0.05$.

for combined data (Table). Slopes are presented in the Table in $\log -\log$ form (i.e., $\mathrm{dB} / 10$ ).

\section{The Relationship between RNFL Thickness and Ganglion Cell Density Estimated from Functional Tests}

Figure 4 shows the relationship between RNFL thickness and estimates of GC density from PGRA ( $\mathrm{GC}_{\mathrm{PGRA}}$; left panels) and DLS ( $\mathrm{GC}_{\mathrm{DLS}}$; right panels). On inspection of each of the panels in the figure, there does not appear to be nonlinearity in the data. Nonlinearity was not statistically significant (cusum, $P>$ 0.05). Heteroskedasticity is evident in the data, however, with a fanning out of data points toward the healthy end of the spectrum, particularly in the inferior field, when GC density was estimated from DLS. The heteroskedasticity was even more marked, showing much larger estimates of GC density, before removal of the two outliers mentioned previously. Less marked heteroskedasticity is seen when RNFL thickness is plotted together with GC $_{\text {PGRA }}$. It appears, on inspection of Figure 4, that the slope is slightly steeper when GC density was estimated

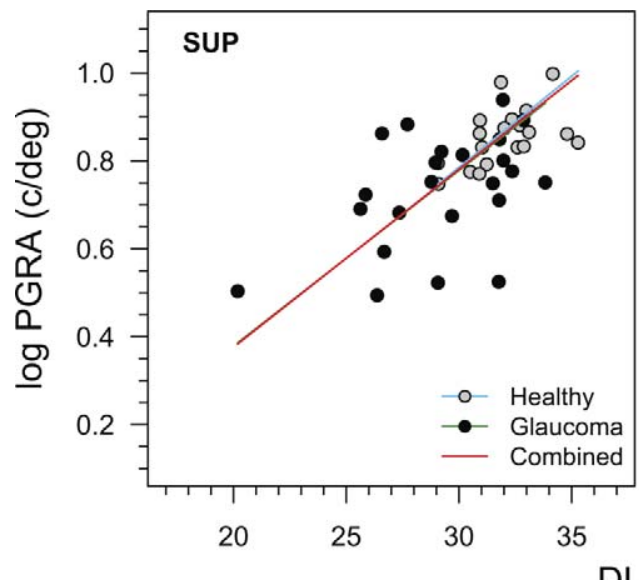

with PGRA than with DLS, particularly in the inferior field. The difference between slopes was not statistically significant in either hemifield following Holm-Bonferroni correction $(P>$ $0.05)$. Figure 4 indicates, on average, a reduction in RNFL thickness of $3.2 \mu \mathrm{m}$ is associated with a loss of $100 \mathrm{GC} / \mathrm{mm}^{2}$, as estimated with PGRA, and a reduction of $1.35 \mu \mathrm{m}$ in RNFL thickness is associated with a loss of $100 \mathrm{GC} / \mathrm{mm}^{2}$, as estimated with DLS. The strength of the association was greater in the superior field (RNFL/GC PGRA $: \tau=0.36$; RNFL/GC $\mathrm{DLS}: \tau=0.34$ ) than in the inferior field (RNFL/GC PGRA $: \tau=0.20 ; \mathrm{RNFL}_{\text {GC }}$ DLS: $\tau$ $=0.19$ ) and appeared similar when either functional measure was used to estimate GC density, at least for the range of damage and sample size studied here.

\section{Agreement between Ganglion Cell Density Estimates from Functional Tests}

Figure 5 shows the agreement between $\mathrm{GC}_{\mathrm{PGRA}}$ and $\mathrm{GC}_{\mathrm{DLS}}$ for both glaucoma patients and healthy subjects, pooled over both hemifields. Agreement was moderate, with marked heteroskedasticity for higher average GC values. The continuous horizontal line represents perfect agreement. CIs are inappro-

FIgURE 3. The association between log PGRA and DLS for glaucoma patients and healthy subjects using Passing-Bablok regression in the superior and inferior hemifields. 

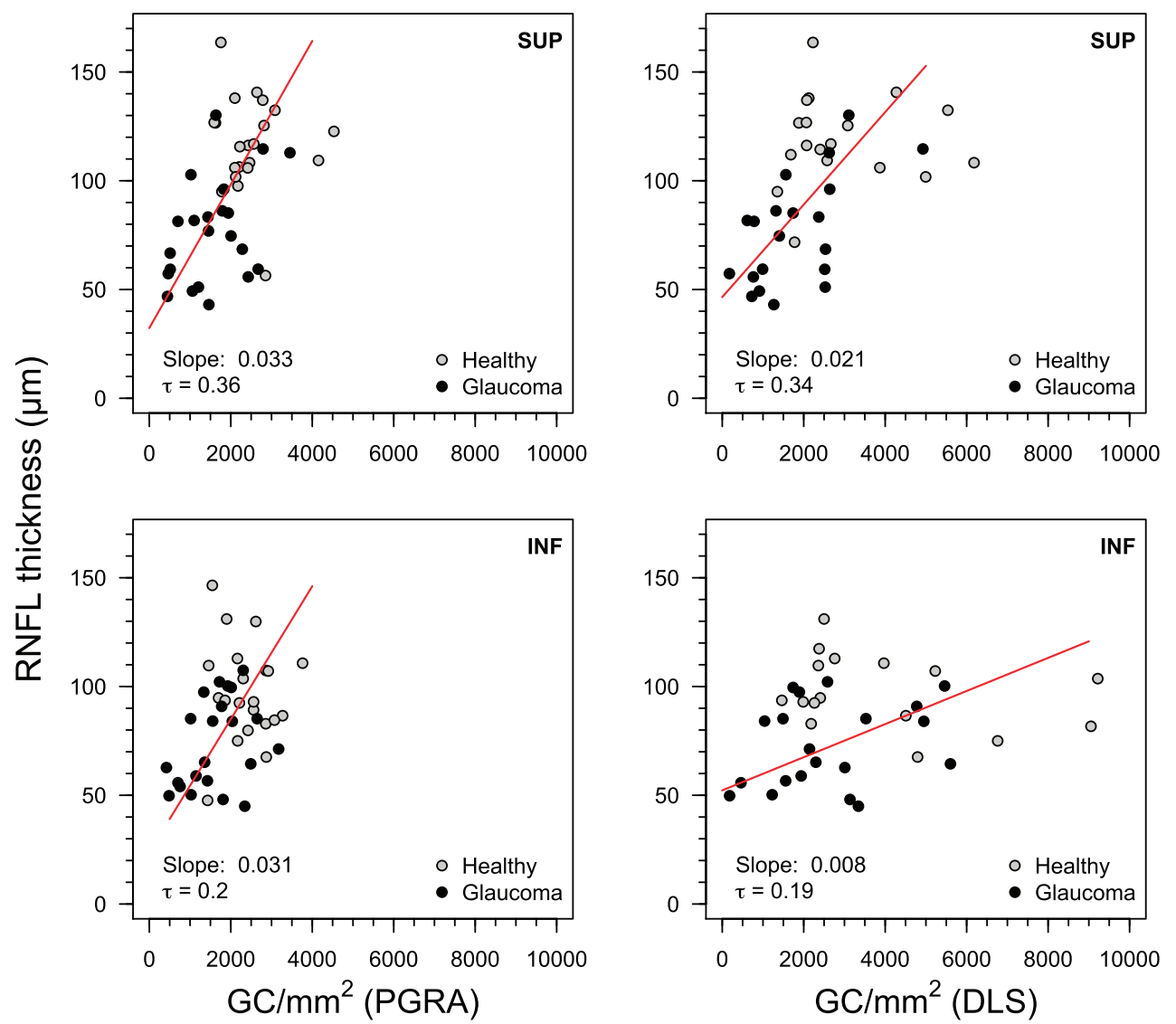

Figure 4. The association between RNFL thickness and GC density estimates in the superior and inferior hemifields from PGRA (left) and DLS (right). Passing-Bablok regression lines are plotted through the combined glaucoma and healthy subject data.

priate in this plot, owing to the heteroskedasticity. It is estimated using the hockey-stick model ${ }^{10}$ that there are 2883 $\mathrm{GC} / \mathrm{mm}^{2}$ for a SAP sensitivity of $31 \mathrm{~dB}$ (the sensitivity at Ricco's area). Figure 5 reveals that below this number, the average difference between estimates by PGRA and DLS is $353 \mathrm{GC} / \mathrm{mm}^{2}$, biased toward DLS estimates. Beyond $2883 \mathrm{GC} / \mathrm{mm}^{2}$ and below $10,000 \mathrm{GC} / \mathrm{mm}^{2}$, however, the average difference between estimates is $4231 \mathrm{GC} / \mathrm{mm}^{2}$, biased toward DLS estimates. Continuous curves drawn in Figure 5 represent the actual error in GC density that would be expected from a $\pm 0.04 \log$ unit or $\pm 1 \mathrm{~dB}$ measurement error in PGRA or DLS, respectively.

\section{Discussion}

The current study is the first to investigate the relationship between PGRA and RNFL thickness in healthy and glaucomatous eyes. Structure/function and function/function relationships are not significantly nonlinear for the range of disease studied here, when measurements are expressed on a log-log scale. While these relationships are not strong, the moderate association is impressive, considering the narrow range of glaucomatous visual field damage (the glaucoma patients had early glaucoma and no dense defects in the test locations). On the other hand, it is worth bearing in mind that the narrow range of damage also reduces the power to make definitive judgments about linearity in the data. Although the structure/ function relationships appear similar on inspection of the data and slopes in Figure 2, and the observed difference between slopes falls within the $95 \%$ confidence limits of the permutation distribution, differences cannot be excluded with our sample size. Contrary to previously published assumptions about the structure/function relationship in healthy observers, ${ }^{11}$ a significant relationship was found, in the current study, between PGRA and RNFL thickness and between DLS and

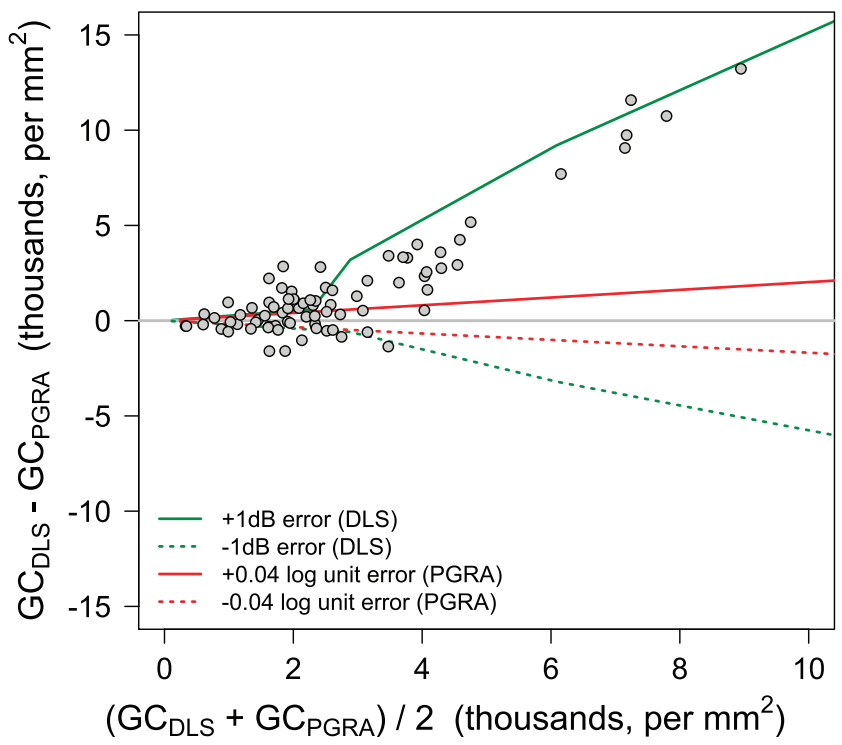

Figure 5. The agreement between estimates of GC density using PGRA and DLS. Red and green lines represent the actual error in GC density for a $\pm 0.04 \log$ unit measurement error by PGRA and a $\pm 1 \mathrm{~dB}$ measurement error by DLS, respectively. 
RNFL thickness in healthy subjects alone, and the differences between these slopes and those for patient data were not statistically significant.

Although the association between RNFL thickness and GC density, as determined by DLS, showed no significant deviation from linearity, the data show heteroskedasticity for estimates of GC density, with a wide range in more normal locations. Figure 5 shows moderate agreement between methods used to estimate GC density, with marked heteroskedasticity and a wide range at the upper end of the abscissa. Factors that likely influence appreciation of the true structure/function relationship as well as lack of perfect agreement between GC estimates here include (1) the accuracy of the objective measurement, (2) the difference between the spatial extent of the stimuli, (3) the difference in stimulus contrast at threshold, and (4) the nature of the psychophysical task.

It is well understood that variability in functional measurements affects appreciation of the true structure/function relationship; however, it is sensible to consider structural measurement variability and interindividual variation in retinal architecture also. Garway-Heath et al. ${ }^{38}$ reported that the between-individual SD of the clock-hour point of entry of a particular axon into the optic disc was $7.2^{\circ}$, so that the entry point varies by almost $\pm 15^{\circ}$ (or $30^{\circ}$ between extremes) in $95 \%$ of individuals. Therefore, averaging RNFL thickness values over $30^{\circ}$ segments is prudent for a reasonable appreciation of the structure/function relationship. However averaging may mask small localized defects. In such instances, a relatively deep perimetric defect might appear inconsistent with an apparently normal or slightly reduced RNFL thickness value in the corresponding peripapillary segment. Measurement errors may be particularly great if they are acquired and/or averaged over a steep RNFL thickness gradient. Small eye movements during the OCT scan acquisition, and small misalignments in the scan circle may also be a source of variability. ${ }^{4}$ Moreover, it is reasonable to suggest that the level of damage at a particular visual field location may be under- or overestimated by OCT, compared with that by functional tests, owing to averaging over GC axons corresponding to more distal regions of the retina, even if scans are acquired at the localized level. Functional tests, however, evaluate the functional integrity of local GCs.

When measuring visual function in glaucoma, the clinician is measuring the visual consequences of retinal GC loss. PGRA and DLS are two such methods. ${ }^{10,27}$ However, tests of PGRA and DLS determine GC function with very different stimuli and test paradigms. It is, therefore, of interest to compare local estimates of GC density (per millimeter squared) from the two functional tests and relate these to RNFL thickness. This approach may also allow for comparison between functional tests, independent of measurement scale and the dynamic range of the instrument. Appreciably, these estimates of GC density may be influenced by any factor that causes the functional measure to be variable. In the current study, the area and presentation duration of the two stimuli, as well as the response criteria, differ greatly. The degree to which the visual system responds to a stimulus depends on the extent of retina covered by the stimulus. The stimulus is firstly sampled by the photoreceptors and GCs at the retinal level and further processed by second stage spatial filters. ${ }^{10,16}$ In order to obtain optimal resolution performance, the grating should contain at least 5 to 6 cycles. ${ }^{46}$ The extent of the PGRA stimulus in the current study was, therefore, much larger than that of the conventional Goldmann III stimulus used in SAP $\left(0.43^{\circ}\right.$ diameter). It has been argued in previous literature that, on one hand, a larger stimulus (whatever its form) pools responses from neighboring retinal areas and yields more information about the functional integrity of densely defective regions in advanced glaucoma. ${ }^{3,47,48}$ On the other hand, it has been argued that smaller stimuli are preferred in the detection of highly localized damage, as one can detect smaller localized areas of functional loss without intrusion by otherwise healthy retina. ${ }^{3,47}$ While it might be that our stimuli measure different attributes of visual function, a similar argument exists here. It is entirely possible that a large stimulus might recruit more neighboring damaged areas than the smaller Goldmann III, especially when one considers the relatively wide spacing between targeted retinal areas in conventional perimetry. ${ }^{49}$ In such a case, PGRA would sample a larger area of retina and may signal a dropout of GCs before conventional measures of DLS. Conversely, the smaller DLS stimulus might allow identification of more specific localized defects, while resolution of the larger grating stimulus may be less vulnerable to heterogeneous (patchy) loss. In such a scenario, although local aliasing might occur within the grating stimulus at an area of heterogeneous loss, a more global veridical percept could mask this; a phenomenon known as supra-Nyquist acuity. ${ }^{50}$ Thus, subtle localized damage may well be missed by PGRA and estimates of GC density may be somewhat overestimated with respect to those estimated using DLS. Also worth considering is that if ganglion cell density declines such that the stimulus window contains fewer than 5 to 6 cycles, GC density may increasingly become underestimated. ${ }^{46}$ In the current study we find that GC density estimated with DLS declines at a slightly greater rate than that estimated with PGRA, for a given rate of decline in RNFL thickness.

If GC dysfunction precedes cell death, it might be argued that the maximum contrast grating stimulus elicits a response from healthy cells together with a proportion of dysfunctional cells that are still capable of relaying a signal from a high contrast stimulus. Indeed, dysfunctional cells would still make up part of the neural element of the RNFL. Similarly, it might be that only healthy cells respond to a small circular increment at threshold and dysfunctional cells are unable to respond to such a dim stimulus. A similar hypothesis, supported by grating acuity data has previously been reported (Demirel S, IOVS 2004; 45:ARVO E-Abstract 3303) and additional experiments are required to explore this further, however. An overestimation of GC density using PGRA might also arise as a result of the two alternative forced choice nature of the resolution task.

The deleterious effects of cataract (both real and simulated) on DLS are well known, ${ }^{51-54}$ and a comparison of the effects of increased intraocular stray light on PGRA and SAP showed that PGRA was more resilient. ${ }^{54}$ In individuals with elevated intraocular stray light, one might, therefore, expect artefactually lower GC estimates for DLS than PGRA overall.

The nonlinear relationship between GC number and sensitivity on a decibel scale, ${ }^{10}$ as well as the large logarithmic step size in the SAP thresholding staircase, may influence the estimation of GC density from DLS here. The hockey stick model ${ }^{10}$ describes the relationship between SAP sensitivity and GC number in a healthy subject with respect to eccentricity. It predicts that when the Goldmann III stimulus is smaller than the critical summation area, there is a 10:1 relationship between SAP sensitivity (in decibels) and GC number (in $\log$ units): a $1 \mathrm{~dB}$ reduction in sensitivity with every $0.1 \mathrm{log}$ unit decline in GC number. When the Goldmann III is larger than Ricco's area, a $0.25 \mathrm{~dB}$ reduction in sensitivity corresponds to a $0.1 \mathrm{log}$ unit decline in GC number. Thus, when the Goldmann III stimulus is larger than Ricco's area, a $1 \mathrm{~dB}$ error in sensitivity leads to a more marked error in the estimate of underlying GC density than a $1 \mathrm{~dB}$ error when the stimulus is smaller. Sensitivity at Ricco's area has been reported to be around 31 $\mathrm{dB},{ }^{10}$ thus, larger errors in GC density are expected for a $1 \mathrm{~dB}$ error when the true sensitivity is greater than or equal to $32 \mathrm{~dB}$ than when it is less than or equal to $30 \mathrm{~dB}$; sensitivity levels 
that may be considered normal for a healthy subject in the central retina. An overestimation of threshold leads to a greater percentage error in GC estimates than an underestimation. In SAP, a $2 \mathrm{~dB}$ step size is employed, therefore, the error in GC estimates would be expected to be even larger. Since DLS in the current study was determined from spatial summation curves, and, thus, measurement variability is reduced, the error in GC density arising from an error in the DLS determination would be expected to be less than that arising from an error in the SAP sensitivity measurement. Of note in Figure 5 is that the curve showing the expected error in GC density for $\mathrm{a}+1 \mathrm{~dB}$ measurement error in DLS closely reflects the heteroskedasticity in the data. Figure 5 also shows the error in GC density arising from a 1 step error in the resolution acuity estimate. This error is not as marked as that arising from a $1 \mathrm{~dB}$ error in DLS.

In conclusion, function/function and structure/function relationships are observed, using both PGRA and DLS as the functional measure, even reaching significance in healthy subjects. Slopes in healthy subjects alone are not significantly different to those observed in data for glaucoma patients. Consideration should therefore be given to the association between structure and function in healthy observers when attempting to improve structure/function models in glaucoma. The associations between RNFL thickness and GC density estimates determined from PGRA and DLS are not significantly nonlinear over the range of damage studied here, however, there is heteroskedasticity in the $\mathrm{RNFL} / \mathrm{GC}_{\mathrm{DLS}}$ data, with overly large estimates of GC density in healthy locations. Agreement between estimates of GC density (using PGRA and DLS) is moderate, but large disagreements occur in locations displaying normal or near normal visual function. The association between PGRA and retinal structure is at least as close as that between DLS and retinal structure in healthy eyes and over the range of glaucomatous visual field damage studied here.

\section{References}

1. Henson DB, Chaudry S, Artes PH, Faragher EB, Ansons A. Response variability in the visual field: comparison of optic neuritis, glaucoma, ocular hypertension, and normal eyes. Invest Ophthalmol Vis Sci. 2000;41:417-421.

2. Artes PH, Hutchison DM, Nicolela MT, LeBlanc RP, Chauhan BC. Threshold and variability properties of matrix frequencydoubling technology and standard automated perimetry in glaucoma. Invest Ophthalmol Vis Sci. 2005;46:2451-2457.

3. Wall M, Woodward KR, Doyle CK, Artes PH. Repeatability of automated perimetry: a comparison between standard automated perimetry with stimulus size III and V, matrix, and motion perimetry. Invest Ophthalmol Vis Sci. 2009;50:974979.

4. Gabriele ML, Ishikawa H, Wollstein G, et al. Optical coherence tomography scan circle location and mean retinal nerve fiber layer measurement variability. Invest Ophthalmol Vis Sci. 2008;49:2315-2321.

5. Zhu H, Crabb DP, Schlottmann PG, Wollstein G, Garway-Heath DF. Aligning scan acquisition circles in optical coherence tomography images of the retinal nerve fiber layer. IEEE Trans Med Imaging. 2011;30:1228-1238.

6. Garway-Heath DF, Caprioli J, Fitzke FW, Hitchings RA. Scaling the hill of vision: the physiological relationship between light sensitivity and ganglion cell numbers. Invest Ophthalmol Vis Sci. 2000;41:1774-1782.

7. Harwerth RS, Carter-Dawson L, Shen F, Smith EL III, Crawford ML. Ganglion cell losses underlying visual field defects from experimental glaucoma. Invest Ophthalmol Vis Sci. 1999;40: 2242-2250.
8. Kerrigan-Baumrind LA, Quigley HA, Pease ME, Kerrigan DF, Mitchell RS. Number of ganglion cells in glaucoma eyes compared with threshold visual field tests in the same persons. Invest Ophthalmol Vis Sci. 2000;41:741-748.

9. Quigley HA, Dunkelberger GR, Green WR. Retinal ganglion cell atrophy correlated with automated perimetry in human eyes with glaucoma. Am J Ophthalmol. 1989;107:453-464.

10. Swanson WH, Felius J, Pan F. Perimetric defects and ganglion cell damage: interpreting linear relations using a two-stage neural model. Invest Ophthalmol Vis Sci. 2004;45:466-472.

11. Hood DC, Kardon RH. A framework for comparing structural and functional measures of glaucomatous damage. Prog Retin Eye Res. 2007;26:688-710.

12. Malik R, Swanson WH, Garway-Heath DF. 'Structure-function relationship' in glaucoma: past thinking and current concepts. Clin Experiment Ophthalmol. 2012;40:369-380.

13. Garway-Heath DF, Holder GE, Fitzke FW, Hitchings RA. Relationship between electrophysiological, psychophysical, and anatomical measurements in glaucoma. Invest Ophthalmol Vis Sci. 2002;43:2213-2220.

14. Malik R, Swanson WH, Garway-Heath DF. Development and evaluation of a linear staircase strategy for the measurement of perimetric sensitivity. Vision Res. 2006;46:2956-2967.

15. Anderson RS. The psychophysics of glaucoma: improving the structure/function relationship. Prog Retin Eye Res. 2006;25: 79-97.

16. Pan F, Swanson WH. A cortical pooling model of spatial summation for perimetric stimuli. $J$ Vis. 2006;6:1159-1171.

17. Johnson CA, Adams AJ, Casson EJ, Brandt JD. Blue-on-yellow perimetry can predict the development of glaucomatous visual field loss. Arch Ophthalmol. 1993;111:645-650.

18. Sample PA, Weinreb RN. Color perimetry for assessment of primary open-angle glaucoma. Invest Ophthalmol Vis Sci. 1990;31:1869-1875.

19. Johnson CA, Samuels SJ. Screening for glaucomatous visual field loss with frequency-doubling perimetry. Invest Ophthalmol Vis Sci. 1997;38:413-425.

20. Quaid PT, Flanagan JG. Defining the limits of flicker defined form: effect of stimulus size, eccentricity and number of random dots. Vision Res. 2005;45:1075-1084.

21. Liu S, Lam S, Weinreb $\mathrm{RN}$, et al. Comparison of standard automated perimetry, frequency-doubling technology perimetry, and short-wavelength automated perimetry for detection of glaucoma. Invest Ophthalmol Vis Sci. 2011;52:7325-7331.

22. Sample PA, Medeiros FA, Racette L, et al. Identifying glaucomatous vision loss with visual-function-specific perimetry in the diagnostic innovations in glaucoma study. Invest Ophthalmol Vis Sci. 2006;47:3381-3389.

23. Metha $\mathrm{AB}$, Lennie P. Transmission of spatial information in Scone pathways. Vis Neurosci. 2001;18:961-972.

24. Beirne RO, Zlatkova MB, Anderson RS. Changes in human short-wavelength-sensitive and achromatic resolution acuity with retinal eccentricity and meridian. Vis Neurosci. 2005;22: 79-86.

25. Dacey DM. The mosaic of midget ganglion cells in the human retina. J Neurosci. 1993;13:5334-5355.

26. Anderson RS, Zlatkova MB, Demirel S. What limits detection and resolution of short-wavelength sinusoidal gratings across the retina? Vision Res. 2002;42:981-990.

27. Thibos LN, Cheney FE, Walsh DJ. Retinal limits to the detection and resolution of gratings. J Opt Soc Am A. 1987; 4:1524-1529.

28. Beirne RO, Logan JF, Zlatkova MB, et al. Peripheral resolution for achromatic and SWS gratings in early to moderate glaucoma and the implications for selective ganglion cell density loss. Invest Ophthalmol Vis Sci. 2003;44:4780-4786. 
29. Thibos LN, Bradley A. New methods for discriminating neural and optical losses of vision. Optom Vis Sci. 1993;70:279-287.

30. Thibos LN. Acuity perimetry and the sampling theory of visual resolution. Optom Vis Sci. 1998;75:399-406.

31. Redmond T, Garway-Heath DF, Zlatkova MB, Anderson RS. Sensitivity loss in early glaucoma can be mapped to an enlargement of the area of complete spatial summation. Invest Ophthalmol Vis Sci. 2010;51:6540-6548.

32. Anderson RS, Wilkinson MO, Thibos LN. Psychophysical localization of the human visual streak. Optom Vis Sci. 1992; 69:171-174.

33. Rovamo J, Virsu V, Laurinen P, Hyvarinen L. Resolution of gratings oriented along and across meridians in peripheral vision. Invest Opbthalmol Vis Sci. 1982;23:666-670.

34. Redmond T, Zlatkova MB, Garway-Heath DF, Anderson RS. The effect of age on the area of complete spatial summation for chromatic and achromatic stimuli. Invest Ophthalmol Vis Sci. 2010;51:6533-6539.

35. Levitt H. Transformed up-down methods in psychoacoustics. $J$ Acoust Soc Am. 1971;49:(suppl 2):467+.

36. Pentland A. Maximum likelihood estimation: the best PEST. Percept Psychopbys. 1980;28:377-379.

37. Seber GAF, Wild CJ. Nonlinear regression. New York: John Wiley \& Sons; 1989.

38. Garway-Heath DF, Poinoosawmy D, Fitzke FW, Hitchings RA. Mapping the visual field to the optic disc in normal tension glaucoma eyes. Ophthalmology. 2000;107:1809-1815.

39. Drasdo N, Fowler CW. Non-linear projection of the retinal image in a wide-angle schematic eye. Br J Opbthalmol. 1974; 58:709-714.

40. Russell RA, Malik R, Chauhan BC, Crabb DP, Garway-Heath DF. Improved estimates of visual field progression using bayesian linear regression to integrate structural information in patients with ocular hypertension. Invest Opbthalmol Vis Sci. 2012;53: 2760-2769.

41. Bablok W, Passing H, Bender R, Schneider B. A general regression procedure for method transformation. Application of linear regression procedures for method comparison studies in clinical chemistry, Part III. J Clin Chem Clin Biochem. 1988;26:783-790.
42. Passing H, Bablok W. A new biometrical procedure for testing the equality of measurements from two different analytical methods. Application of linear regression procedures for method comparison studies in clinical chemistry, Part I. J Clin Chem Clin Biochem. 1983;21:709-720.

43. Bland JM, Altman DG. Statistical methods for assessing agreement between two methods of clinical measurement. Lancet. 1986;1:307-310.

44. Holm S. A simple sequentially rejective multiple test procedure. Scand J Stat. 1979;6:65-70.

45. R Development Core Team. R: A Language and Environment for Statistical Computing. Vienna, Austria: R Foundation for Statistical Computer; 2005.

46. Anderson RS, Evans DW, Thibos LN. Effect of window size on detection acuity and resolution acuity for sinusoidal gratings in central and peripheral vision. J Opt Soc Am A Opt Image Sci Vis. 1996;13:697-706.

47. Fellman RL, Lynn JR, Starita RJ, Swanson WH. Clinical importance of spatial summation in glaucoma. In: Heijl A, ed. Perimetry Update 1988/1989. Amsterdam: Kugler \& Gedini; 1989:313-324.

48. Swanson WH, Felius J, Birch DG. Effect of stimulus size on static visual fields in patients with retinitis pigmentosa. Opbthalmology. 2000;107:1950-1954.

49. Anderson DR, Patella VM. Automated Static Perimetry. St. Louis, MO: Mosby; 1999.

50. Wang YZ, Bradley A, Thibos LN. Aliased frequencies enable the discrimination of compound gratings in peripheral vision. Vision Res. 1997;37:283-290.

51. Budenz DL, Feuer WJ, Anderson DR. The effect of simulated cataract on the glaucomatous visual field. Opbthalmology. 1993;100:511-517.

52. Carrillo MM, Artes PH, Nicolela MT, LeBlanc RP, Chauhan BC. Effect of cataract extraction on the visual fields of patients with glaucoma. Arch Opbthalmol. 2005;123:929-932.

53. Lam BL, Alward WL, Kolder HE. Effect of cataract on automated perimetry. Opbthalmology. 1991;98:1066-1070.

54. Anderson RS, Redmond T, McDowell DR, Breslin KM, Zlatkova MB. The robustness of various forms of perimetry to different levels of induced intraocular stray light. Invest Ophthalmol Vis Sci. 2009;50:4022-4028. 